\title{
A Precision Evaluating Method of DEM Using Map Spots Generated by Contour Superposition
}

\author{
Zechun Huang*, Zhu Xu, Qianning Zhang, Min Xiao, Zipu Liu, Yongyou Qian and Tianting He \\ Faculty of Geosciences and Environmental Engineering, Southwest Jiaotong University, Chengdu, Sichuan, 611756, China \\ ${ }^{*}$ Corresponding author
}

\begin{abstract}
Aiming at the shortcomings of traditional contour superposition analysis in quantitative evaluation of DEM error, the purpose of this paper is to propose a new method to measure the DEM accuracy by using the map spots of contour superposition. To begin with, the concepts of the difference rate and the matching rate for contour overlaying was described, and the calculating methods about the two indices were illustrated in accordance with the map spots generated by contour overlaying. Then, this paper comparatively illustrated the consistency between the terrain coincidence reflected by the matching rate of the same name contour lines and the mean elevation difference of the reconstructed DEM and the original DEM. Finally, this paper analyzed the influence of contour interval to the difference rate of the contour superposition. Meanwhile, taking slope as the complexity factor of terrain and selecting different experimental areas with different average slope, the correlation between the difference rate of contour superposition and the average slope of experimental area was quantitatively measured by regression analysis method. The experimental results show that map spots generated by contour superposition can be used to reflect the terrain coincidence between reconstructed DEM and original DEM, and the matching rate of contours is positively correlated with the total number of the map spots, but negatively correlated with the relevant indexes of the area of the map spots. The method proposed in this paper not only can reflect the statistical characteristics of DEM error, but also can describe the spatial distribution of error.
\end{abstract}

Keywords-DEM; contour superposition; map spot; error analysis; regression analysis

\section{INTRODUCTION}

DEM error affects the reliability and accuracy of digital terrain analysis results, so the study of DEM data quality has very important theoretical significance and application value [1] In practical application, we should not only consider the elevation precision of DEM, but also study the coincidence between the terrain expressed by reconstructed DEM and natural terrain [2]. Usually the contour superposition method is used to evaluate the terrain coincidence [3]. But using this method can only qualitatively analyze the general situation of DEM precision and the terrain coincidence. Thus, the traditional contour superposition method lacks quantitative measurement criteria for evaluation of DEM error [3, 4].

To improve the effect of traditional contour superposition method which cannot quantitatively describe the accuracy of DEM, the academia has carried on the related exploration, and has obtained some research achievements. The concept of terrain description error was proposed to quantitatively describe the terrain expression accuracy of DEM [5]. According to that the change of the terrain surface shape is necessary to change the contour shape, the method of evaluating the contour superposition error which reflect the accuracy of the terrain surface shape was presented [6]. Using the ratio of the area difference between reconstructed contour and original contour to the length of original contour as an index for DEM precision evaluation, scholars illustrated the overall deviation of before and after reconstructing contour and the coincidence degree between the reconstructed terrain and original terrain [7]. Based on two precision indexes of the maximum reconstructed offset and average maximum reconstructed offset, the coincidence degree between DEM and actual terrain was measured in the local area [8]. In addition, for the sake of evaluating the agreement degree of the river net, a quantitative description method was produced by using the ratio of the total area of small polygon generated by overlaying of two river networks and the area of the basin in experimental area [9].

Described as above, a comparative analysis of contour reconstructed by DEM is a visual quality evaluating method, and it can quantitatively analysis the coincidence degree between the reconstructed terrain surface and the original terrain surface, furthermore it can evaluate the quality of DEM [6]. However, these methods only use a single index to evaluate the overall accuracy of DEM. So, they can neither describe the statistical characteristics of contour superposition errors, nor describe its spatial distribution quantitatively. For this purpose, this paper aims to propose a new method to evaluate the accuracy of reconstructed DEM using map spots generated by contour superposition. We will build correlation indices of map spots, and then analyze the statistical characteristics of reconstructed DEM error by means of statistical description method. It is hoped that not only can quantitatively evaluate the coherence degree between the reconstructed terrain surface and the actual terrain surface, but also can describe the statistical characteristics and spatial distribution of the errors of the reconstructed DEM.

\section{Dem ERror Model BASED ON MAP SPOTS OF CONTOUR SUPERPOSITION}

The basic idea of the DEM error model is to carry out the spatial overlaying operation based on the contour from reconstructed DEM and the original DEM, and then to generate a series of map spots as shown in Figure I, and to reflect quantitatively the difference degree of the two contours generated from reconstructed DEM and original contour according to the area of the map spots. The ratio of all the map 
spots total area and the experimental region area is called the difference rate, which indicated the whole difference between the reconstructed contour and the original contour. The calculating formula of the indices is shown in equation (1). The calculating formula for matching ratio between the reconstructed contour and the original contour is shown in equation (2). In the equation (1) and (2), $A$ represents the area of the experimental region, and $\alpha_{i}$ represents the area of the map spot $i$, and $n$ indicates the number of map spots, and $\varepsilon$ indicates the difference radio of contour superposition, and $p$ stands for the matching ratio of contour superposition.

$$
\begin{aligned}
& \varepsilon=\frac{\sum_{i=1}^{n} \alpha_{i}}{A} \\
& p=1-\varepsilon
\end{aligned}
$$

\section{EXPERIMENTAL METHODS}

For accessing DEM accuracy by using the map spots of contour superposition, this paper firstly takes the conversion of DEM from $30 \mathrm{~m}$ resolution to $90 \mathrm{~m}$ resolution as an example, then selects four kinds of method which are the nearest neighbor resampling, mode resampling, median aggregating, and mean aggregating to rebuild DEM; Secondly, the two contour lines generated according to the reconstructed DEM and the original DEM are overlapped to create map spots through spatial calculating of polygon, and moreover analyze the spatial distribution characteristics of map spots. Thirdly, the mean elevation difference index is taken as the reference object of the terrain coincidence degree, and the consistency of the coincidence rate between the contour difference rate and the mean elevation difference index will be analyzed. Based on the four kinds of scale transformation method, the statistical analysis of the indexes of the map spots will be carried out, and the relationship between the contour matching rate and the index variables of the map spots will be explored.

The density of contour lines can affect the number of map spots, which will lead to variety of DEM error distribution. For contour interval can affect the density of contour lines, it is necessary to analyze the influence of the contour interval on contour matching rate. To this end, firstly based on the data of one experimental area, we use the nearest neighbor resampling method to reconstruct DEM from $30 \mathrm{~m}$ resolution into $90 \mathrm{~m}$ resolution. Secondly, for the reconstructed DEM and the original DEM, the corresponding contour lines will be created respectively by six kinds of contour interval of $50 \mathrm{~m}, 100 \mathrm{~m}$, $200 \mathrm{~m}, 300 \mathrm{~m}, 400 \mathrm{~m}, 500 \mathrm{~m}$. Meanwhile, the correlation index value of the map spots will be calculated successively by spatial overlapping operation of contour lines with the same name. Finally, the relationship between the contour lines and the number of map spots, the area index, the difference rate and the matching rate will be analyzed to find out the influence rule of the contour interval to the DEM error evaluated by map spots of contour overlapping.

The slope is chosen as the terrain feature factor to explore whether the method of evaluating the DEM error by map spots is affected by the terrain features. Firstly, this paper selects 16 experimental areas of different terrain complexity, using the nearest neighbor resampling method for DEM scale transformation, and calculate the indexes value of the average slope, contour difference rate and contour matching rate. Then, the correlation between the difference rate and the average slope will be analyzed by using the scatter plot, and the correlation coefficient will be used to analyze the correlation degree between them. Finally, the regression analysis method will be used to quantitatively describe the correlation between them, and the quantitative relationship between the contour difference rate and the slope of the experimental area will be measured with empirical regression equation.

\section{Statistical ANAlysis of MAP SPOTS AND ThE EFFECT OF CONTOUR INTERVAL}

The mean elevation difference index reflects the terrain coincidence between the reconstructed DEM and the original DEM. The area of map spots can also be used to measure the deviation degree of the contour with the same altitude, and it can be used to quantitatively evaluate the matching degree between the two contour lines generated respectively by the reconstructed DEM and the original DEM. The statistical data of map spots are listed in Table I. Through the comparative analysis of the difference between height mean difference and the contour difference ratio, it could be seen that the smaller absolute value of the mean elevation difference is, the smaller the contour difference rate is, and vice versa. The statistical results showed that the map spots of contour superposition can be used to reflect the terrain coincidence degree of reconstructed DEM. Analyzing the statistical data of map spots, it showed that contour matching rate and the total number of map spots was positively correlated, and it was negatively correlated with the total area, mean area, area standard deviation.

TABLE I. STATISTICAL DATA OF MAP SPOTS GENERATED BY CONTOUR SUPERPOSITION USING DIFFERENT METHODS

\begin{tabular}{|l|l|r|r|r|r|}
\hline \multicolumn{2}{|l|}{ Methods to reconstruct DEM } & Nearest neighbor resampling & Median aggregating & Mean aggregating & Mode resampling \\
\hline \multicolumn{2}{|l|}{ number of map spots } & 48276 & 44504 & 33456 & 18618 \\
\hline \multirow{3}{*}{$\begin{array}{l}\text { Area of map spots } \\
{\left[\mathrm{m}^{2}\right]}\end{array}$} & Sum & 28628668.8 & 31956977.8 & 37806971.3 & 112994271.6 \\
\cline { 2 - 6 } & Mean & 593.0 & 718.1 & 1130.1 & 6069.1 \\
\cline { 2 - 6 } & $\begin{array}{l}\text { Standard } \\
\text { deviation }\end{array}$ & 1080.8 & 1329.2 & 1967.3 & 25908.4 \\
\hline \multicolumn{2}{|l|}{ Mean height difference [m] } & 0.8 & 1.1 & 1.2 & -11.8 \\
\hline \multicolumn{2}{|l}{ Difference rate [\%] } & 3.2 & 3.5 & 4.2 & 12.5 \\
\hline \multicolumn{2}{|l}{ Matching rate [\%] } & 96.8 & 96.5 & 95.8 & 87.5 \\
\hline
\end{tabular}


As shown in table II, with the increase of the contour interval, the number of the map spot reduced. At the same time, the difference rate decreased, and the matching rate increased. In the same experimental area, the smaller the contour interval is, the more the contour lines generated by DEM is, and the larger the total area of map spots is, and the greater the difference rate is, but the smaller the matching rate is. Further analysis results showed that the contour interval was negatively correlated with the difference rate and the number of map spots, and the correlation between the contour interval and the contour matching rate was positively correlated. From the above analysis, contour interval has an influence on the evaluation of the DEM accuracy. Therefore, it is necessary to make a comparative analysis with the same contour interval when the method is used to measure the terrain surface coincidence.

TABLE II. STATISTICAL DATA OF THE CONTOUR INTERVAL INFLUENCE TO THE MAP SPOTS

\begin{tabular}{|c|c|c|c|c|c|c|c|c|}
\hline \multirow{2}{*}{$\begin{array}{c}\text { contour } \\
\text { interval(m) }\end{array}$} & \multirow{2}{*}{$\begin{array}{l}\text { number of } \\
\text { map spots }\end{array}$} & \multicolumn{5}{|c|}{ Area of map spots $\left(\mathrm{m}^{2}\right)$} & \multirow{2}{*}{$\begin{array}{c}\text { Difference } \\
\text { rate }(\%)\end{array}$} & \multirow{2}{*}{$\begin{array}{l}\text { Matching } \\
\text { rate (\%) }\end{array}$} \\
\hline & & minimum & maximum & sum & mean & Std. & & \\
\hline 50 & 88524 & 0.000005 & 22580.0 & 57265227.0 & 646.9 & 1170.7 & 6.4 & 93.6 \\
\hline 100 & 42941 & 0.000005 & 24138.5 & 28858774.7 & 672.1 & 1219.6 & 3.2 & 96.8 \\
\hline 200 & 21356 & 0.000034 & 27586.5 & 14381969.8 & 673.4 & 1236.0 & 1.6 & 98.4 \\
\hline 300 & 14272 & 0.000007 & 21864.1 & 9436242.2 & 661.2 & 1229.1 & 1.0 & 99.0 \\
\hline 400 & 10554 & 0.000034 & 21977.5 & 7135833.8 & 676.1 & 1207.1 & 0.8 & 99.2 \\
\hline 500 & 8520 & 0.000041 & 13270.4 & 5752290.4 & 675.2 & 1230.3 & 0.6 & 99.4 \\
\hline
\end{tabular}

\section{Correlation Between the Contour Difference RATE AND The Average SLOPE}

The sample data contain the average slope and the contour difference rate, which were calculated based on the DEM of 16 experimental areas. Results of statistical analysis shows that with the increase of the average slope, the difference rate increases, and the contour matching rate decreases. As shown in Figure II, it could be seen that approximate linear relationship between the difference rate and average slope is obvious. For quantitatively determining the correlation between them, the correlation analysis of the average slope and the difference rate were carried out. The correlation coefficient of the two variables was 0.96 . The value of correlation coefficient showed that the difference rate was correlated with slope. To further determine the degree of influence between the two variables, the average slope was taken as the independent variable, and the difference rate was used as the dependent variable, and a linear regression model was established based on independent variables and dependent variables. On the base of the sample data, the model parameters were solved, and the empirical regression equation (3) is obtained. In the regression equation (3), Slope stand for the average slope of experiment area, and $\varepsilon$ stand for difference rate of contour superposition.

$$
\varepsilon=0.094308 \times \text { Slope }+0.244789
$$

$\mathrm{R}^{2}$ is the deterministic coefficient of the regression equation, which represents the explanatory power of the independent variable in the equation. The $\mathrm{R}^{2}$ value calculated by the regression model is 0.9139 , which shows that the "average slope" explains the variation degree of the difference rate of $91.39 \%$. From the empirical regression equation, when the average slope increased by 1 degree, the difference rate will increase by 9.4308 percentage points. The results showed that the influence of the average slope on the contour difference ratio was not significant.

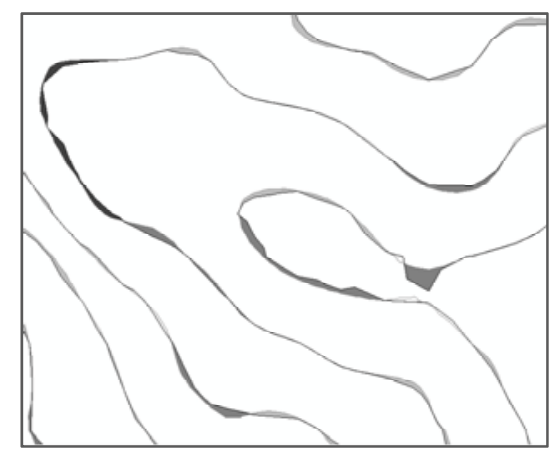

FIGURE I. MAP SPOTS OF CONTOURS SUPERPOSITION

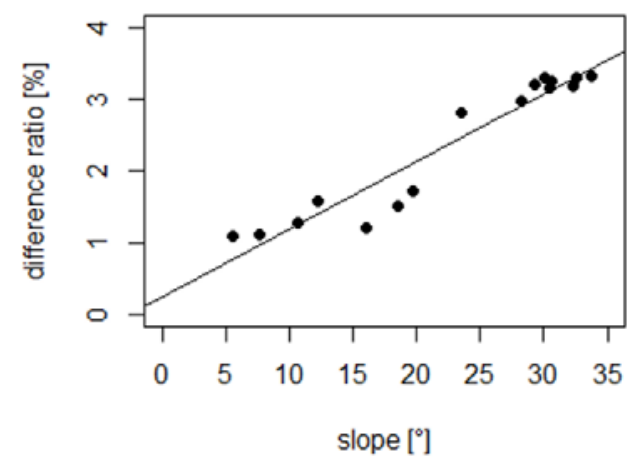

FIGURE II. CORRELATION BETWEEN THE DIFFERENCE RATE AND THE AVERAGE SLOPE

\section{SUMMARY}

Aiming at shortcomings of the traditional contour matching in automatic interpretation and quantitative evaluation, this paper proposed a new method of evaluating DEM error, which was evaluated by using the ratio of the map spots and the total area of the experimentation area. This method overcame the shortcomings of the traditional contour superposition method which only carry out qualitative analysis. It can not only 
quantitatively evaluate the DEM error, but also can describe its statistical characteristics. The experimental results showed that using contour matching rate to describe the terrain coincidence degree of the reconstructed DEM had the same effect compared with using the average elevation difference index. Under the condition of using the same contour interval to create contour lines, the contour matching rate was positively correlated with the number of the map spots, but negatively correlated with the area indexes of the map spots. There was a strong linear correlation between the difference rate of contour matching and the average slope of the experiment area. Compared with the traditional contour reconstruction method, this method proposed in this paper not only can access quantitatively the DEM error overall, but also can according to the area of map spots in different position analyze the spatial distribution of DEM error. Contour interval had a major influence on contour matching rate in the same experiment area. Therefore, it is necessary to compare and analyze terrain matching effect using map spots in the case of the same contour interval. But the selecting strategy of contour interval needs further study.

\section{ACKNOWLEDGEMENT}

This research was financially supported by Mapping Geographic Information Public Service Industry Research Funds (Grant No.201512028) and the Central Universities Fundamental Research Funds (Grant No.2682014CX017).

\section{REFERENCES}

[1] ZHIWEI W. The study and application of DEM error model based on the reconstructed contours [D]; PLA Information Engineering University, 2007.

[2] CHUN W, YANG T, DUN-XIN J, et al. Study on the accruracy of regulary grid DEM topographical description morphology [J]. Geomatics World, 2008, (01): 46-52+72.

[3] FEN Q, XUE-JUN J. Study on buffer superposition to quantitatively evaluate DEM precision completely [J]. Acta Agriculture Borealioccidentalis Sinica, 2008, 17(03): i336-42.

[4] GUANG-XIA W, SHU-LI B, YIN-BAO Z. Evaluating DEM accuracy based on reconstructed contours [J]. Journal of Geomatics Science and Technology, 2010, 27(01): 9-13.

[5] YONG Z, GUO-AN T, QI P. A mathematical simulation of DEM terrain representation error- a case study in the Loess hill-gully areas of China [J]. Journal of Moutain Science, 2003, 21(02): 252-6.

[6] GUO-AN T, YANG T, CHUN W. Contour-matching-difference and Its application in DEM quality assessment [J]. Bulletin of Surveying and Mapping, 2007, (07): 65-7.

[7] FAN J. Researches on the surface modeling and the accuracy evaluation of DEM [D]; PLA Information Engineering University, 2006.

[8] CHANGQING Z, ZHIWEI W, HAIYAN L. Accuracy Evaluation Model of DEM Based on Recontructed Contours [J]. Geomatics and Information Science of Wuhan University, 2008, 33(02): 153-6.

[9] WEI-TAO L, CHUN W, PENG L, et al. Influences of DEM generalization methods on extracting accuracy of hydrological structure information [J]. Bulletin of Soil and Water Conservation, 2012, 32(04): 168-71. 